19 Revue d'histoire du XIXe siècle

Société d'histoire de la révolution de 1848 et des

révolutions du XIXe siècle

$62 \mid 2021$

Ancêtres

\title{
Introduction. Ancêtres « fin-de-siècle »
}

Nicole Edelman et Louis Hincker

URL : https://journals.openedition.org/rh19/7454

DOI : $10.4000 /$ rh 19.7454

ISSN : $1777-5329$

Éditeur

La Société de 1848

Édition imprimée

Date de publication : 20 juin 2021

Pagination : 9-22

ISSN : 1265-1354

Référence électronique

Nicole Edelman et Louis Hincker, «Introduction. Ancêtres « fin-de-siècle » », Revue d'histoire du XIXe siècle [En ligne], 62 | 2021, mis en ligne le 17 août 2021, consulté le 26 août 2021. URL : http:// journals.openedition.org/rh19/7454; DOI : https://doi.org/10.4000/rh19.7454 


\section{NICOLE EDELMAN, LOUIS HINCKER}

\section{Introduction \\ Ancêtres "fin-de-siècle»}

En 1994, Gérard Namer soulignait, dans sa postface très fournie à la réédition des Cadres sociaux de la mémoire de Maurice Halbwachs ${ }^{1}$, à quel point les années 1880-1918 avaient été une période durant laquelle la mémoire devint en Europe un objet de réflexion et d'étude. Nombreux furent les domaines traversés par une inquiétude mémorielle, considérée au cœur des difficultés que semblait rencontrer le monde occidental en pleine transformation. Gérard Namer proposait de lire cette hantise de la mémoire comme une réponse antithétique à la modernité rationaliste, comme une forme de désenchantement face aux illusions du progrès démocratique et scientifique. Les années qui précédèrent la Grande Guerre virent le développement dans le monde intellectuel d'une nécessaire solidarité entre les vivants et les morts pour parer aux excès de l'individualisme, alors vu de manière négative car synonyme de destruction de lien social. En France, à titre d'exemples, ce fut le cas aussi bien du côté de Barrès en littérature dans Les Déracinés, que du côté de Durkheim en sciences humaines dans Les Formes élémentaires de la vie religieuse. Les recherches sur la mémoire s'imposaient à travers la naissance de la psychologie comme discipline, cherchant comme la jeune neurologie à comprendre ses mécanismes. La philosophie et la littérature s'en emparaient, Zola construisait les Rougon-Macquart sur la mémoire de l'hérédité, Bergson interrogeait les liens entre matière et mémoire, Proust identifiait le phénomène de la réminiscence comme un symptôme du sentiment de finitude. Dans ce contexte, les religions chrétienne et juive invoquaient de manière redoublée «la foi de nos pères» qui implique un rapport à la tradition, une exigence de transmission d'une génération à la suivante et un exercice de mémoire. Telle était la fonction possiblement inclusive de la mémoire jusqu’à lui donner une fonction civique normative face au pessimisme critique finde-siècle.

Le dossier proposé ici entend se concentrer sur les enjeux culturels et intellectuels de la relation entre mémoire, ancestralité, et hérédité durant

1. Maurice Halbwachs, Les Cadres sociaux de la mémoire, Paris, Albin Michel, 1994 [1 $1^{\mathrm{re}}$ éd. 1925], p. 297-366. 
la seconde moitié du XIX siècle, particulièrement dans l'aire culturelle occidentale. En effet, les ancêtres, réels ou imaginés, figurations symboliques de l'obsession mnésique des contemporains, sont alors conviés en bien des endroits pour assumer toutes sortes de tâches : structurer des liens entre générations, reconfigurer des identités, conjurer les spectres d'une décadence ou d'un déclin, construire des récits nationaux, etc. Tantôt leur convocation divise, reclasse et élève des barrières entre les vivants et les morts, tantôt elle unifie et (re)crée une communauté. Cette quête d'ancêtres, bons ou mauvais, marque ce moment en Europe d'abord puis par rebonds dans une grande partie du monde. C'est pourquoi ce dossier propose aussi un plongeon dans le Japon de l'ère Meiji et ses mythes fondateurs de l'ancêtre impérial (Yusuke Inenaga), ainsi qu'aux États-Unis dans le territoire des Lakotas/Sioux avec ses morts victimes des massacres du XIXe siècle (Thomas Grillot). Les conquêtes coloniales, les guerres meurtrières, le libéralisme et l'impérialisme économique déstabilisent en effet alors des traditions religieuses et des relations de pouvoir bien au-delà du seul continent européen, obligeant à retrouver des mythes oubliés pour reconstruire des liens cassés par ces incursions.

Les enjeux politiques sont parfois les mêmes lorsque, après les années 1870, la plupart des États, occidentaux en premier lieu, s'inventent une identité nationale ancrée dans un passé réel ou imaginé. Les Français apprennent que "nos ancêtres, c'est nous dans le passé» et que "si nous ne ressemblons guère à nos pères les Gaulois ${ }^{2}$ ", ils furent quand même - Vercingétorix en tête - des héros et des braves! Par contrecoup, les Juifs de France, comme on le verra dans ce dossier, face à l'antisémitisme puissant de ces décennies, s'opposent à une représentation qui les désigne comme extérieurs à la nation pour au contraire démontrer leur insertion par la mise au jour de documents généalogiques les ancrant dans le substrat national (Mathias Dreyfuss).

Cette quête des ancêtres généralisée s'inscrit largement dans une recherche de reconstruction de liens perdus ou pensés tels, de communautés d'êtres humains. Cette volonté est mue par la crainte d'une décadence de la civilisation occidentale, voire d'une dégénérescence de la race blanche, qui semble mettre en péril cette marche en avant promise ${ }^{3}$. Au début des années 1890, Gustave Le Bon déplore la perte de "l'âme de la race ${ }^{4}$ » et annonce l'effondrement de la civilisation, préparé par la dégénérescence de l'humanité européenne. Le monde savant dans son ensemble, anciennes et nouvelles disciplines observent ce malaise et tentent d'en comprendre les raisons en se tournant vers le passé humain, proche ou lointain.

2. Ernest Lavisse, Histoire de France. Cours élémentaire, livre 1, Paris, Armand Colin, 1913; Étienne Bourdon, La Forge gauloise de la nation: Ernest Lavisse et la fabrique des ancêtres, Lyon, ENS Éditions, 2017.

3. Voir la polémique, en 1894-1895, sur «la faillite de la science», dans l'article : Ferdinand Brunetière, "Après une visite au Vatican", Revue des Deux Mondes, vol. 127, 1895/1, p. 97-118.

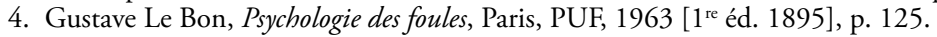




\section{L'HÉRÉDITÉ-DÉGÉNÉRESCENCE COMME RETOUR DES ANCÊTRES}

Développons précisément dans ses détails, et jusque dans ses conséquences durables, la manière par laquelle en France - puis par connexion en Europe -, l'hérédité morbide devient l'objet de recherches médicales à partir du milieu du XIX ${ }^{e}$ siècle ${ }^{5}$. Bien des maladies sont considérées comme héréditaires : la tuberculose, l'alcoolisme, l'hystérie, tout comme les caractères intellectuels et moraux (génie mis à part). L'hérédité devient ainsi l'objet de recherches médicales et le Traité philosophique et physiologique de l'hérédité naturelle de Prosper Lucas, publié en 1847 et en 1850, donne les premières réponses. Dorénavant, les médecins procèdent à des interrogatoires précis de leurs malades sur la santé de leurs parents, grands-parents, fratries et collatéraux proches, cependant rarement au-delà, les ascendants étant dans l'ensemble trop peu connus pour fournir une réponse satisfaisante. Le Dictionnaire encyclopédique des sciences médicales, publié en 1888, n'a pas d'entrée «ancêtre» mais il en est beaucoup question dans l'article "hérédité» qui porte la marque d'un darwinisme interprété. Les travaux de Charles Darwin ont, en effet, profondément modifié le regard sur l'hérédité en le portant vers les débuts de l'humanité et l'article du dictionnaire souligne "la puissance de l'hérédité ancestrale», en particulier pour le maintien des différences entre les races. Il exprime la crainte de l'atavisme chez l'homme «qui reproduit parfois certains caractères qui semblent bien rappeler les plus anciens ancêtres animaux ceux que les transformistes nous donnent pour de très anciens aïeux et dont notre évolution embryologique nous retrace en abrégé la série ${ }^{6} »$.

À la date de parution du dictionnaire, une grande transformation des conceptions de l'hérédité est due aussi à la publication en 1857 du Traité des dégénérescences physiques, intellectuelles et morales de l'espèce humaine et des causes qui produisent ces variétés maladives, écrit par l'aliéniste Bénédict Augustin Morel (1809-1873). La question de la dégénérescence devient désormais partout en Europe une sorte d'obsession débattue et argumentée jusqu'à la guerre de 1914. Les interrogations se déploient vers les causes de ce désastre biologique : d'où vient-il? Est-il occasionnel? Héréditaire? Est-il le symptôme de la décadence de l'homme occidental?

Morel est un aliéniste nommé après la révolution de 1848 dans le grand asile de Maréville (Meurthe-et-Moselle) puis, en mai 1856, comme médecin chef de l'asile de Saint-Yon près de Rouen, grande ville industrielle, où il demeure jusqu'à sa mort. C'est en observant les fous de son asile, qui sont

5. On peut se reporter pour un cadre global à Jean Borie, Mythologie de l'hérédité au XIXe siècle, Paris, Galilée, 1981; Daniel Pick, Faces of Degeneration. A European Disorder, c.1848-c.1918, Cambridge, Cambridge University Press, 1989.

6. Amédée Dechambre (dir.), Dictionnaire encyclopédique des sciences médicales, Paris, Libraires Masson et Asselin, 1888, article «Hérédité», p. 596. 
tous issus des classes ouvrières les plus pauvres de la région, qu'il théorise cette notion de dégénérescence. Morel est un catholique et un créationniste monogéniste - la race humaine dérivant d'un type primitif commun -, les premiers ancêtres sont donc Adam et Ève, créés par Dieu dans leur perfection, et sa foi le rend optimiste : l'humanité ne peut pas périr car ses destinées sont

celles que la sagesse de Dieu lui a fixées, on ne comprend pas que le mot dégénérescence puisse être appliqué dans son acception rigoureuse à l'espèce humaine tout entière. D'après notre définition, la dégénérescence est la déviation maladive d'un type normal primitif, or le progrès, qui est le but de l'humanité est incompatible avec une situation pareille. Nous savons d'un autre côté que si les individus meurent, que si les sociétés disparaissent, il ne s'ensuit pas que l'espèce ne soit immuable?

Son ami Philippe Buchez, rendant compte de l'ouvrage dans les Annales médico-psychologiques, partage cette certitude et souligne que l'idée de dégénérescence est "corrélative à l'idée de perfectibilité et à celle-là seulement ${ }^{8}$ ". Cependant, pour corriger cette déchéance de quelques-un.e.s et empêcher qu'elle ne prenne plus d'ampleur, Morel pense qu'il faut modifier les conditions de vie et de travail des classes ouvrières : "L'exercice de professions dangereuses ou insalubres, l'habitation dans des centres trop populeux ou malsains soumettent l'organisme à de nouvelles causes de dépérissement et conséquemment de dégénérescence ${ }^{9} »$. L'homme est alors

modifié par les conditions mauvaises que lui fait la vie de fabrique, l'exploitation de certains produits qui le mettent en contact avec des émanations toxiques ou qui le forcent à passer la plus grande partie de son existence dans les entrailles de la terre. Si l'on joint maintenant à ces mauvaises conditions générales, l'influence si profondément démoralisatrice qu'exercent la misère, le défaut d'instruction, le manque de prévoyance, l'abus de boissons alcooliques et les excès vénériens, l'insuffisance de la nourriture, on aura une idée des circonstances complexes qui tendent à modifier d'une manière défavorable les tempéraments de la classe pauvre ${ }^{10}$.

En attendant ces transformations, le dégénéré

devient de plus en plus incapable de remplir sa mission dans l'humanité [...] le progrès intellectuel déjà enrayé dans sa personne se trouve encore menacé dans

7. Bénédict Augustin Morel, Traité des dégénérescences physiques, intellectuelles et morales de l'espèce humaines et des causes qui produisent ces variétés maladives, Paris, Baillière, 1857, p. 361.

8. Philippe Buchez, "Rapport fait à la société médico-psychologique sur le traité des dégénérescences physiques, intellectuelles et morales de l'espèce humaine et des causes qui les produisent », Annales médico-psychologiques, t. 1, 1857, p. 459.

9. Bénédict Augustin Morel, Traité des dégénérescences, op. cit., p. 50.

10. Ibid. 
celle de ses descendants $[\ldots]$ il devient non seulement incapable de former dans l'humanité la chaîne de transmissibilité d'un progrès, mais il est encore l'obstacle le plus grand à ce progrès, par son contact avec la partie saine de la population ${ }^{11}$.

Toutefois cette dégénérescence ne compromet pas l'avenir de l'humanité à long terme puisque d'une part le dégénéré devient infertile au bout de quelques générations, d'autre part le métissage des races peut pallier ce déclin ponctuel; et Dieu demeure le maitre de sa création.

Tous ne partagent pas cet optimisme, la notion de dégénérescence faisant florès ${ }^{12}$, l'inquiétude se développe et l'aliéniste Valentin Magnan (18351916), médecin chef du récent asile Sainte-Anne à Paris, défend l'existence d'un nouveau type d'homme, «le dégénéré», différent de l'homme normal. Il estime (comme Morel) que les dégénérescences sont

inhérentes à l'état même de la société qui les engendre. L'inégalité des conditions créée par les besoins intenses de la lutte pour vivre, la misère, l'alcoolisme, le surmenage, l'insalubrité des professions, les agglomérations urbaines, la sophistication des produits alimentaires, les maladies épidémiques et contagieuses, etc., etc., sont autant de maux qu'il appartient à la société sinon de supprimer complètement tout au moins d'atténuer. Si le progrès, chose paradoxale, enfante les causes de la décadence, il faut qu'il sache, en revanche, en multiplier les remèdes $[\ldots]$. La dégénérescence [...] est un mal et un péril social, il importe de lui imposer une hygiène sociale rigoureuse ${ }^{13}$.

Ces dégénérés ne doivent pas devenir les ancêtres de l'humanité future car si cette pathologie peut être d'origine accidentelle, elle peut être aussi d'origine héréditaire comme certains le pensent.

C'est la thèse du médecin italien Cesare Lombroso (1835-1909) ${ }^{14}$ qu'il expose dans L'Uomo delinquente en 1876 (L'Homme criminel, traduit en français en 1887). Il fait du criminel un dégénéré, un produit de l'atavisme, un être chez qui réapparaissent des caractères de bestialité qui se seraient manifestés chez l'un de ses ancêtres. Vingt ans plus tard, renvoyant vers la "découverte récente» de son aide et élève Luigi Roncoroni, il affirme "que la fine anatomie du cerveau des criminels reproduit les caractères des verté-

11. Ibid., p. 5-6.

12. Il est certain que, dans ces années 1890 , le concept de dégénérescence est à son apogée. En 1892, Max Nordau publie Dégénérescence, traduit en français dès 1894, qu'il dédie à «M. le professeur Lombroso ", dans lequel le phénomène est qualifié de "fin-de-siècle». En 1893, André Sanson fait paraître L'Hérédité normale et pathologique. L'ouvrage de Charles Féré, La Famille névropathique. Théorie de l'hérédité et de la prédisposition morbide et de la dégénérescence est de 1894. Le livre Dégénérés et déséquilibrés de Jules Dallemagne est édité en 1895, simultanément en France et en Belgique. On pourrait citer bien d'autres livres encore et des thèses de médecine sur ce thème.

13. Valentin Magnan, Georges Albert Legrain, Les Dégénérés (état mental et syndromes épisodiques), Paris, Rueff, 1895, p. 235.

14. Marc Renneville, «La réception de Lombroso en France (1880-1900)», in Laurent Mucchielli (dir.), Histoire de la criminologie française, Paris, L'Harmattan, 1995, p. 107-135. 
brés inférieurs ${ }^{15}$ ». Naissent ainsi des lignées de criminels et plus largement de "déchets sociaux", de "nuisibles" de toutes sortes. Lombroso donne de l'ampleur à ses travaux en étudiant les femmes, il affirme que dans la race humaine, la femme égale ou surpasse l'homme avant la puberté puis se laisse distancier, dépasser et « reste en arrière [...] preuve d'un phénomène atavique commun aux races inférieures : la précocité ${ }^{16}{ }^{\prime}$. Et il conclut que la femme est moins souvent criminelle que l'homme mais que l'équivalent de la criminalité innée est, chez elle, la prostitution et dans ses interminables descriptions, il retrouve bien souvent et souligne le retour d'un atavisme. Lombroso ne convainc pas, loin de là, ses collègues et les débats et les controverses entre médecins sont nombreux dans les revues et les congrès - un Lacassagne, la chose est bien connue, regarde du côté de la causalité sociologique.

\section{PAR-Delà la dégénérescence : L'anCestralité fondatrice}

Dans ce désaccord, il faut faire une place à l'hypothèse originale des spirites, dissonante mais partagée largement par les croyants de cette nouvelle religion. En 1857, son créateur Allan Kardec affirme que le spiritisme est la troisième révélation divine après celle de Moïse et du Christ, faite par des esprits extraterrestres et adaptée aux temps modernes du XIX ${ }^{\mathrm{e}}$ siècle; c'est une «religion scientifique» puisqu'elle procède dans son élaboration comme les sciences positives en s'appuyant sur les expériences successives des tables tournantes, de l'écriture automatique et des incarnations médiumniques qui toutes étaient, selon lui, contrôlables et renouvelables ${ }^{17}$. Pour les spirites, la planète Terre tout entière est un lieu d'expiation pour la réincarnation d'esprits ayant commis des fautes dans des vies antérieures, mieux située que Mars où se réincarnent les esprits qui ont à se racheter plus encore mais moins bien que les autres planètes plus proches du soleil. Parler de déclin n'a donc pas grand sens, les hommes et les femmes vivent pour se racheter, progresser et surtout ne pas se réincarner sur notre planète. En revanche, Léon Denis, un des successeurs de Kardec, maintient une des croyances essentielles en la réincarnation qui modifie radicalement la relation traditionnelle aux ancêtres en détruisant la filiation biologique, puisque la mère et le père ne donnent à l'enfant que l'enveloppe charnelle qui reçoit, au moment précis de la naissance et du premier souffle, un esprit qui vient d'ailleurs ${ }^{18}$. Celui-ci peut s'incarner dans ce bébé qui vient de naitre pour expier des fautes de sa

15. Cesare Lombroso, La Femme criminelle et la prostituée Grenoble, Jérome Million, 1991 [ $1^{\text {re }}$ éd. 1895 et traduit en français dès 1896], préface, p. 29.

16. Ibid., p. 28.

17. Nicole Edelman, Voyantes, guérisseuses et visionnaires en France, Paris, Albin Michel, 1995; id., Histoire de la voyance et du paranormal. Du XVIII siècle à nos jours, Paris, Le Seuil, 2006.

18. Léon Denis, Après la mort. Exposé de la doctrine des esprits, Paris, Librairie des sciences psychologiques, 1893. 
vie antérieure ou parfois exceptionnellement pour aider les habitant.e.s de la terre à progresser et terminer ainsi son parcours incarné avant de devenir un esprit sans corps. La conception spirite s'oppose donc radicalement à celle du monde médical qui parle d'hérédité et de dégénérescence. Les parents d'un «mauvais» esprit ne sont responsables ni d'un dégénéré, ni d'un délinquant, ni même d'un monstre, ils ont pour rôle de le guider vers une amélioration qui lui permettra de progresser dans ses futures réincarnations. À l'inverse, l'esprit supérieur en mission sur terre peut participer à la régénération de sa famille d'accueil ou d'un groupe social plus large. Si les spirites partagent la notion d'hérédité, ils proposent donc des solutions à la fois optimistes et déculpabilisantes.

L'importance de l'hérédité est, en effet, d'autant plus un leitmotiv de cette fin de siècle que les découvertes de Charles Darwin se diffusent, que l'on soit en accord avec lui ou pas. Le débat touche à l'anthropologie générale ${ }^{19}$ et la seconde moitié $\mathrm{XIX}^{\mathrm{e}}$ siècle rebat totalement les cartes du débat idéologique sur l'explication des origines. À ce moment, l'ancestralité devient objet d'une réflexion globale, intellectuelle, scientifique, métaphysique. Boucher de Perthes, un des fondateurs de la préhistoire, à la recherche des origines de l'humanité, affirme l'existence d'un homme antédiluvien ${ }^{20}$ qui allonge alors démesurément l'histoire de l'être humain (Fanny Defrance-Jublot). Or, ce très vieil ancêtre ne ressemble sans doute pas au premier Adam de la Bible, d'autant que Jean-Baptiste de Lamarck (1744-1829) a proposé l'idée d'une transformation des corps, appuyée sur une théorie transformiste de l'évolution du vivant. François Jacob a expliqué que «jusqu'au milieu du XIXe siècle, le monde vivant représentait un système à régulation externe. Qu'ils soient figés depuis la création ou qu'ils aient progressé par une succession d'événements, les êtres organisés constituaient toujours une série continue des formes ${ }^{21}$ ». En revanche, avec la naissance de la biologie et surtout avec les découvertes de Charles Darwin «disparaît l'idée d'une harmonie préconçue par quoi serait imposé un système de relations aux êtres organisés. [...] Aucun individu d'une espèce n'est plus identique aux autres ${ }^{22}$ ». La question de l'hérédité se pose alors pleinement, et, par conséquent, celle de la transmissibilité de qualités et de défauts, de tares et de malformations, jusqu'à l'échelle de chaque famille. Dans La Filiation de l'homme et la sélection liée au sexe $e^{23}$ que

19. Sur la réception de Darwin en France : Liv Grjebine, «Le Darwinisme en débat dans la société française (1859-1900). Le rôle du débat public dans la légitimation d'une théorie scientifique», thèse de doctorat d'histoire, sous la direction de Bruno Belhoste, Université Paris 1 Panthéon-Sorbonne, 2018.

20. Jacques Boucher de Perthes, De l'Homme antédiluvien et de ses auvres, Paris, Librairies JungTreuttel, Derache, Dumoulin, V. Didron, 1860.

21. François Jacob, La Logique du vivant, une histoire de l'hérédité, Paris, Gallimard, coll. «Tel», 1995, p. 190.

22. Ibid., p. 191

23. Charles Darwin, La Filiation de l'homme et la sélection liée au sexe, traduction coordonnée par Michel Prum (Institut Charles Darwin International), Paris, Éditions Syllepse, 2000. La traduction du 
Darwin publie en 1871 et qui complète ses propos développés dans L'Origine des espèces par l'exposé de l'évolution de l'homme lui-même, l'auteur affirme que «l'homme doit sa naissance à une longue lignée d'ancêtres ${ }^{24}$ » qui part des amphibiens en passant par les mammifères dont les Simiidés, puis les singes. "Nos ancêtres ont divergé de la souche des Catarhiniens ${ }^{25}$ " qui sont une des divisions des Simiidés. Un long cheminement dont Darwin retrace les progrès puisque toutes les nations civilisées sont bien les descendantes de barbares. "L'homme s'est élevé, bien qu’à pas lents et interrompus, à partir d'une humble condition jusqu'au plus haut niveau par lui jamais atteint dans la connaissance, la morale et la religion ${ }^{26}{ }^{\prime}$. L'homme blanc européen du XIx e siècle est une sorte de sommet de l'évolution et Darwin explique le ralentissement de ce progrès en Europe par différents facteurs liés à notre mode de vie et de pensée protégeant les plus faibles ce qui ouvre la voie aux interprétations d'Herbert Spencer et au darwinisme social.

Pour Darwin, le progrès est indéniable, nos ancêtres ont d'abord vécu en petites hordes.

En regardant assez loin en arrière dans le cours du temps, et jugeant à partir des habitudes sociales de l'homme tel qu'il existe à présent, l'opinion la plus probable est qu'il a vécu originellement en petites communautés, chacun avec une seule épouse, ou s'il était puissant avec plusieurs, qu'il gardait jalousement contre tous les autres hommes. Ou bien il a pu ne pas être un animal social, et cependant vivre avec plusieurs épouses, comme le gorille, car tous les indigènes "sont d'accord sur le fait qu'on ne voit qu'un seul mâle adulte dans une bande; quand le jeune mâle grandit, un affrontement a lieu pour la suprématie, et le plus fort en tuant et en chassant les autres, s'établit en chef de la communauté" (Dr Savage). Les jeunes mâles, en étant ainsi expulsés et condamnés à l'errance, ont dû empêcher quand ils réussissaient enfin à trouver une partenaire, des entrecroisements trop proches dans les limites de la même famille ${ }^{27}$.

Le tabou de l'inceste aurait donc permis de sortir de la violence et du droit du plus fort. Des anthropologues confirment cette hypothèse, le double essai Social Origins de Andrew Lang et Primal Law de James Jasper Atkinson, publié en 1903, reprend cette affirmation que les hommes ont commencé par former des groupes très restreints qui chacun édictèrent des interdits, l'un des principaux étant le mariage entre hommes et femmes du même groupe ${ }^{28}$.

titre The Descent of Man par La Filiation de l'homme paraît la plus juste; "Descent" signifie "descendre de», procéder d'une même série d'ancêtres.

24. Ibid., p. 255.

25. Ibid., p. 245.

26. Ibid., p. 234.

27. Ibid., p. 711.

28. Chroniqué par Émile Durkheim dans L'Année sociologique (1896/1897-1924/1925), vol. 7, 1902, p. 407-411. 
Interdit pour lequel Atkinson avance la raison d'une jalousie sexuelle de l'homme dominant le groupe, qui serait donc à l'origine de cette prohibition de l'inceste.

Cette conception s'inscrit dans celle plus large du rôle des croyances primitives, celles de nos premiers ancêtres dans l'évolution humaine. Des anthropologues s'intéressent à ce qu'ils vont nommer le totémisme, observé dans des territoires récemment colonisés - l'Australie en particulier. Retrouver les premières formes de vie et de croyances communes des primitifs devrait permettre de comprendre le processus de civilisation, ses écueils et son cheminement jusqu'à nos jours. Émile Durkheim participe à cette recherche, inquiet de la "phase d'ébranlement profond" que traversent nos sociétés qui "sont obligées de se renouveler et se cherchent laborieusement, douloureusement ${ }^{29}$ ». Pour lui, "les indigènes de l'Australie [...] ne représenteraient pas seulement un état social simple, mais l'état social primitif par lequel toute l'humanité a dû passer. Si l'on admet ce postulat, on comprend l'intérêt immense que présentent ces sauvages; c'est chez eux qu'il faut aller pour retrouver les formes primitives de la famille, de l'art, de la science ${ }^{30}$ ».

Un grand nombre d'ouvrages sont écrits sur ce thème, ceux de l'Écossais James George Frazer (1854-1941) en particulier, qui publie en 1887 Totemism, une première étude d'une centaine de pages, qu'il complète en exposant en 1910 la somme de tous les contextes totémiques connus dans Totemism and Exogamy. A Treatise on Certain Early Forms of Superstition and Society ${ }^{31}$. Émile Durkheim leur donne à son tour une place nouvelle et centrale dans la sociologie qu'il conceptualise ${ }^{32}$. Il en publie les résultats en 1912 dans Les Formes élémentaires de la vie religieuse, le système totémique en Australie. Il y développe l'idée que le totémisme est un type représentatif de la religion primitive dans un processus de civilisation.

Passé le cap du siècle, l'émergence et la diffusion de la psychanalyse participent alors notoirement de cette inquiétude face au passé de l'humanité et à ce qui en reste dans chaque homme et femme. À la veille de la Grande Guerre, Freud prolonge en même temps qu'il exacerbe tout le débat qui le précède ${ }^{33}$. Dans Totem et tabou, publié en 1913, avec pour sous-titre Quelques concordances dans la vie d'âme des sauvages et des névrosés, Freud, à son tour,

29. Émile Durkheim, «L'avenir de la religion. Le sentiment religieux à l'heure actuelle, troisième entretien : la conception sociale de la religion", Paris, Vrin, 1914, p. 97-105, qui est le texte d'une communication faite à la séance du 18 janvier 1914 de l'Union de libres penseurs et de libres croyants pour la culture morale.

30. Paul Descamps, "Le totémisme chez les indigènes de l'Australie», Bulletin de la Société internationale de science sociale (Paris), 27 (96), septembre 1912, p. 105b-110b, p. 105.

31. James George Frazer, Totemism and Exogamy. A Treatise on Certain Early Forms of Superstition and Society, Londres, Macmillan and Co, 1910, 4 vol.

32. Sur la réception de Frazer en France : Myron Achimastos, «Durkheim et Frazer : un débat sur le totémisme", L'Année sociologique, vol. 62, 2012/2, p. 313-327.

33. L'histoire de sa "réception" qui, au départ, tient du rejet dans le milieu sociologique et anthropologique français a été faite : Alain de Mijolla, Freud et la France, 1885-1945, Paris, PUF, 2010; Antoine Compagnon, Céline Surprenant (dir.), Freud au Collège de France, 1885-2016, Paris, 
se "propose de soumettre le problème du totémisme à une étude spéciale, en faisant appel à la méthode psychanalytique ${ }^{34}$ ». Il veut "créer un lien entre ethnologues, linguistes, folkloristes, etc., d'une part, et psychanalyste de l'autre ${ }^{35}$ ". Son souci est celui d'un médecin qui veut comprendre certaines névroses obsessionnelles qu'il rencontre dans sa pratique, il pense qu'il existe une ressemblance dans la peur et la culpabilité en cas d'omission entre le cérémonial névrotique de ses patient.e.s et les actes sacrés du rite religieux. Le totémisme, en tant que forme de croyance ancestrale, paraît lui offrir un espace pour rechercher des origines à ces sentiments de peur et de culpabilité. Il se penche à son tour sur ces habitants primitifs de l'Australie déjà étudiés, considérant que ces peuples sauvages ou semi-sauvages ont une vie psychique qui «constitue une phase antérieure bien conservée de notre propre développement ${ }^{36}$ ».

L'argumentation de Totem et tabou s'appuie sur de très nombreuses références prises dans des livres d'anthropologues, d'archéologues de spécialistes des religions ou de médecin, Wilhelm Wundt, en particulier, qui a écrit plusieurs volumes sur la psychologie des peuples. Ces lectures et son analyse conduisent Freud à proposer une première hypothèse, celle de la substitution du père à l'animal totémique :

Si l'animal totémique n'est autre que le père, nous obtenons [...] ceci : les deux commandements capitaux du totémisme, les deux prescriptions tabou qui en forment comme le noyau, à savoir la prohibition de tuer le totem et celle d'épouser une femme appartenant au même totem coïncident quant à leur contenu, avec les deux crimes d'Edipe, qui a tué son père et épousé sa mère, et avec les deux désirs primitifs de l'enfant dont le refoulement insuffisant ou le réveil forment peut-être le noyau de toutes les névroses ${ }^{37}$.

Puis à partir de l'ouvrage de William Roberton Smith sur la religion des Sémites paru en $1889^{38}$, Freud conclut que «la mise à mort et l'absorption périodique du totem aux époques ayant précédé le culte de divinités anthropomorphiques formaient un élément très important de la religion totémique ${ }^{39}$ ". Manger le totem ensemble renforcerait l'identité du groupe et lui donnerait la vie sacrée dont le totem est l'incarnation. Freud confronte alors cette hypothèse d'abord avec celle du darwinisme, Darwin n'accorde bien sûr aucune place au totémisme dans ses travaux mais il suppose «un père violent, jaloux,

Collège de France, 2018 [https://books.openedition.org/cdf/5660], [https://books.openedition.org/ cdf/5669] (liens consultés en mars 2021).

34. Sigmund Freud, Totem et tabou, Paris, Petite bibliothèque Payot, 1970, p. 12, note 2.

35. Ibid., p. 6.

36. Ibid., p. 9.

37. Ibid., p. 152 .

38. L'ouvrage de Smith provoqua une grande controverse en France, voir le compte rendu à sa réédition en 1970 : Jean Hadot, Archives de sciences sociales des religions, $\mathrm{n}^{\circ}$ 30, 1970, p. 241.

39. Sigmund Freud, Totem et tabou, op. cit, p. 159. 
gardant pour lui toutes ses femelles et chassant ses fils au fur et à mesure qu'ils grandissent ${ }^{40}$ ». Puis Freud propose la sienne, confirmée par celle de l'anthropologue James Jasper Atkinson : «Un jour, les frères chassés se sont réunis, ont tué et mangé le père, ce qui mit fin à la horde paternelle ${ }^{41}$.» Un sentiment de culpabilité les saisit qui engendre les deux tabous fondamentaux du totémisme, l'inceste qui seul permet aux frères de vivre ensemble, et l'animal totémique, substitut du père, qu'il est interdit de tuer, décision qui calme la culpabilité du meurtre initial et qui peu à peu est oublié.

Pour la psychanalyse, totémisme et exogamie ont donc une origine commune et simultanée. Partant de cette période ancestrale et de ses hypothèses psychanalytiques, Freud mène la réflexion jusqu'à nos jours, l'idéal du père transforme l'animal totem en homme qui devient un dieu, «avec l'institution de divinités paternelles, la société privée de père s'est transformée peu à peu en société patriarcale. La famille est devenue une reconstitution de la horde primitive ${ }^{42}$ ». Dans le christianisme, "dans le mythe chrétien, le péché originel résulte incontestablement d'une offense envers Dieu le père. Or le Christ a libéré les hommes du poids du péché originel, en sacrifiant sa propre vie, nous sommes en droit de conclure que ce péché avait consisté dans un meurtre ${ }^{43}$ \%. Le crime à expier ne peut être que le meurtre du père, la doctrine chrétienne avoue donc sa culpabilité dans l'acte criminel des premiers ancêtres. La responsabilité de ce crime originel a persisté pendant des millénaires et s'est transmise de génération en génération tandis que le souvenir de la faute s'effaçait. Mais, finalement, peut-être même que ce crime n'a jamais eu lieu! En effet, Freud par son expérience clinique a pu s'apercevoir que le sentiment de responsabilité du névrosé ne reposait pas sur une réalité matérielle mais sur une réalité psychique. Il aurait donc pu en aller de même chez les peuples primitifs qui auraient seulement ressenti cette pulsion meurtrière et cannibale à l'égard du père, de "l'ancêtre primitif ${ }^{44}$ " sans jamais passer à l'acte. Cependant, Freud incline plutôt pour croire à la réalité de ce meurtre primitif.

Cette conviction argumentée de l'existence d'un meurtre primitif et de sa culpabilité est un noyau fort de la pensée freudienne sur l'origine des croyances religieuses. $S^{\prime}$ il en vient à comparer la religion avec une sorte de névrose universelle, c'est par sa réflexion de médecin thérapeute face à des hommes et des femmes en souffrance psychique. Mais il va au-delà, il pense aussi en juif, certes athée mais connaissant l'antisémitisme, particulièrement violent à Vienne après l'élection du maire antisémite Karl Lueger en 1897 (après trois refus de l'empereur François Joseph). Il approfondit ainsi son étude en l'orientant vers le christianisme, puis le judaïsme et à la fin de sa vie interprète le récit biblique à

40. Ibid., p. 162.

41. Ibid., p. 163 et la note 2 sur Atkinson.

42. Ibid., p. 171.

43. Ibid., p. 177.

44. Ibid., p. 183. 
propos de Moïse qui aboutit à la publication, en 1939, de L'Homme Moïse et la religion monothéiste, également une analyse de l'antisémitisme. Freud pense qu'à l'origine du judaïsme, il y a aussi peut-être un parricide : celui de Moïse commis par les Juifs. Il pose l'hypothèse qu'un haut dignitaire égyptien ou un prêtre au nom proche de Moïse, croyant à la religion monothéiste du dieu Aton, libère une tribu sémite tombée en esclavage et leur impose cette religion. Ce peuple se révolte et Moïse est assassiné. Puis le meurtre est oublié. Et bien plus tard à la rencontre à Quadés en Palestine avec d'autres tribus qui avaient pour Dieu un certain Yahvé, une fusion se réalisa entre Yahvé et le Dieu de Moïse. Freud pose donc à nouveau à l'origine des religions le postulat du meurtre fondateur : celui de Moïse, promoteur d'une religion monothéiste, puis d'une autre façon celui de Jésus, pour payer le meurtre originel, crime que Paul transforme en péché originel racheté par le sacrifice d'une victime et donc avoué. Mais une partie des Juifs n'adoptant pas la nouvelle doctrine chrétienne, ils sont accusés plus tard d'avoir mis Dieu à mort. "Responsabilité tragique; ils ont eu à l'expier lourdement ${ }^{45}$.» C'est l'antisémitisme toujours renaissant.

Près d'un siècle de controverses sur le recours aux ancêtres et le retour sur ce passé primitif prend ainsi sa source dans une inquiétude du présent, il s’agit pour certains d'apaiser la crainte d'une décadence de la civilisation occidentale et d'un arrêt du progrès en en cherchant les causes. On peut mesurer le chemin parcouru. Partout dans le monde occidental, la seconde moitié du xix ${ }^{e}$ siècle noue donc un rapport nouveau avec le temps, à l'aune les réflexions philosophiques, des développements rapides des sciences. Il ne s'agit plus seulement d'une relation à un passé révolu, conséquences des révolutions politiques, de l'obsolescence de l'Ancien Régime en son temps balisé par les généalogies des princes et des rois, déterminé par l'attente de l'apocalypse et de l'Au-delà promis par la doctrine chrétienne et la Bible. C'est l'attente du Progrès - avec une majuscule - dans lequel les hommes s'engagent inéluctablement, qui se trouve totalement repensé. Les travaux anthropologiques et ceux de Darwin sur ces hommes qui sont regardés comme l'équivalent de nos ancêtres permettent de donner de l'espoir, de rassurer en montrant que le Progrès reste possible (mais pas certain) pour les hommes blancs du monde occidental puisque le totémisme et le tabou de l'inceste auraient permis aux premières hordes de vivre côte à côte sans s'entre-tuer. En revanche, les médecins sont plus partagés et moins optimistes, et l'angoisse de la dégénérescence est encore omniprésente en psychiatrie durant l'entre-deux-guerres. Quant aux conclusions de Freud elles demeurent - sur les plans tant historiques que psychanalytiques - inaudibles pour beaucoup ou inacceptables et donc sans effet sauf, peut-être, pour ceux et celles qui purent être analysés et rassérénés par une cure avec lui ou l'un de ses confrères.

45. Sigmund Freud, L'Homme Moïse et la religion monothéiste, Paris, Gallimard, coll. «Folio essais», 1986, p. 243. 
Le dossier que l'on va lire entend souligner que le thème d'une dette à l'égard des ancêtres, voire d'une culpabilité, ou encore d'une relation de solidarité entre les générations, n’est nullement réservé aux religions polythéistes en opposition aux religions universalistes, ni comme étant une spécificité des sociétés dites exotiques, ni non plus comme spécifique de notre actuelle mode de la généalogie ou encore des recherches des racines dans les sociétés postcoloniales, et postindustrielles ${ }^{46}$. N'est-ce pas le propre des sociétés en pleine mutation que de s'interroger sur les ancêtres, de les rechercher alors que tout s'accélère en donnant le sentiment que les repères les mieux établis disparaissent? Le xix ${ }^{e}$ siècle mérite d'être regardé aussi à ce titre. Les processus d'ancestralisation sont des constructions dynamiques, indices de mondes en mouvement. C'est bien plutôt l'excès de ruptures qui sollicite et galvanise les dispositifs du rapport fictif à certains morts choisis, porteurs d'autorité. La tradition anthropologique a depuis longtemps identifié la fonction cathartique du culte mortuaire ${ }^{47}$ : la séparation des morts et des vivants, à l'aide de rituels appropriés, passe par la reconnaissance à terme de certains d'entre eux comme des ancêtres, tous n'accédant pas à ce statut.

Ceux-là sont donc des constructions sociales instables, qui touchent aux reconfigurations des identités, à la transmission entre générations, au rapport qu'une société entretient avec son passé, au traitement social et culturel des morts, au processus de subjectivation. Cependant, ces derniers nécessitent un temps de reconnaissance et un investissement rituel de la part des vivants afin de bénéficier d'une revitalisation symbolique. Il est entendu que ces reconfigurations identitaires s'incarnent dans des pratiques de mémorisation, de commémoration et de patrimonialisation qui peuvent déborder les seules limites lignagères familiales, utiles pour tout groupe qui prétend se distinguer des autres. C'est pourquoi, on pourra considérer plusieurs processus de construction de l'ancestralité, cohabitant ou en concurrence, dans une même aire culturelle. L'invention des ancêtres entre dans le répertoire des ressources pour revendiquer reconnaissance et droits.

Tout autant, l'inquiétude mémorielle symptomatique de la période sur laquelle se concentre notre dossier, et dont les travaux d'Halbwachs se font encore l'écho durant l'entre-deux-guerres ainsi que le développement du darwinisme et du freudisme, témoigne d'une hantise qui n’aura pas nécessai-

46. Sur les transformations - nullement son atténuation - du paradigme généalogique dans les sociétés contemporaines, se reporter au numéro de la revue d'anthropologie Civilisations (vol. 63, 2014/1-2) ayant pour thème L'ancestralité revisitée, ou encore au volume collectif dirigé par deux historiennes : Isabelle Luciani, Valérie Piétri, L'Incorporation des ancêtres : généalogie, construction $d u$ présent (du Moyen Âge à nos jours), Aix-en-Provence, Presses universitaires de Provence, 2016.

47. Robert Hertz, "Contribution à une étude sur la représentation collective de la mort", L'Année sociologique, vol. 10, 1905-1906, p. 48-137; Jack Goody, Death, Property and the Ancestors. A Study of the Mortuary Customs of the Lodagaa of West Africa, Stanford, Stanford University Press, 1962. 
rement trouvé d'issue apaisée. On peut faire l'hypothèse que le phénomène s'étend à des domaines multiples de la vie sociale, bien au-delà de ses traductions scientifiques et littéraires que notre dossier a tenté de mettre en avant. Nous ne faisons que suggérer d'aller rechercher dans ses multiples variations, au sein des milieux sociaux les plus divers, si effectivement un sentiment du déclin active une sensibilité à l'ancestralité, particulièrement dans la seconde moitié du XIX siècle. En considérant que les ancêtres sont paradoxaux : tantôt recours face au malheur et à l'infortune, tantôt malédiction entravant les destinées. Ils peuvent être revendiqués ou subis, correspondre à une volonté de renforcement de l'héritage ou inversement d'évitement. Aux ancêtres ressources s'opposent les ancêtres pathogènes. À leurs propos, comme par leur intermédiaire, se renouent les différences entre le prescrit et l'interdit, et donc la construction sociale des normes. Les reconnaittre aide à chasser les fantômes (les morts ennemis); les dé-crypter permet d'établir une collectivité commune aux morts et aux vivants; ainsi s'édifient les systèmes institutionnels fondés sur le principe de la parenté. Il semble difficile d'échapper à cette injonction qui s'impose dans ce paysage mental fin-de-siècle en Europe occidentale. Victor Hugo conçoit ainsi un fauteuil pour les ancêtres dans sa salle à manger de Hauteville House, sur lequel personne n'a le droit de s'asseoir, réservé qu'il est aux «absents qui sont présents» - "ABSENTES ADSUNT» est-il gravé sur le meuble visible aujourd'hui -, sans doute pour rappeler sa croyance en une survie de l'esprit. Dans ses carnets, le projet faisait apparaitre un monument fantomatique, entre l'ébauche et la ruine, typique de l'univers de ses multiples dessins. Mais on verra que Stéphane Mallarmé tente malgré tout de défier cette présence oppressante, en regardant l'ancêtre tel un fétiche de ce qui reste d'une croyance perdue, cherchant une voie pour déjouer les dispositifs d'énonciation organisant la société contemporaine dans tout ce qui les enracine et les ordonne généalogiquement (Louis Hincker).

Notre dossier étend, enfin, la réflexion aux ancêtres du XIXe siècle réinventés ultérieurement. Leur destinée symbolique nous semble devoir être considérée comme partie prenante d'une histoire du XIX ${ }^{\mathrm{e}}$ siècle qui perdure en se survivant à lui-même et pas seulement comme une configuration du temps présent. L'invention des ancêtres - jamais donnée à l'avance, aucun répertoire fixe n'étant à portée de main, toujours à renégocier donc - est une modalité des sociétés qui identifient leurs transformations comme autant profitables que contraignantes. C'est donc un récit sur les Lakotas/Sioux des États-Unis, qui ouvre un dialogue entre périodes historiques, en termes de descendance et d'héritage différés, de perte et de retrouvailles, de survie et de recréation, qui nous sert ici à le dire.

Nicole Edelman est maîtresse de conférences honoraire en histoire contemporaine à l'Université de Paris Ouest Nanterre

Louis Hincker est professeur d'histoire contemporaine à l'Université Clermont-Auvergne 